\title{
How voluntary actions modulate time perception
}

\author{
Dorit Wenke $\cdot$ Patrick Haggard
}

Received: 27 November 2008 / Accepted: 6 May 2009 / Published online: 27 May 2009

(c) The Author(s) 2009. This article is published with open access at Springerlink.com

\begin{abstract}
Distortions of time perception are generally explained either by variations in the rate of pacing signals of an "internal clock", or by lag-adaptation mechanisms that recalibrate the perceived time of one event relative to another. This study compares these accounts directly for one temporal illusion: the subjective compression of the interval between voluntary actions and their effects, known as 'intentional binding'. Participants discriminated whether two cutaneous stimuli presented after voluntary or passive movements were simultaneous or successive. In other trials, they judged the temporal interval between their movement and an ensuing tone. Temporal discrimination was impaired following voluntary movements compared to passive movements early in the action-tone interval. In a control experiment, active movements without subsequent tones produced no impairment in temporal discrimination. These results suggest that voluntary actions transiently slow down an internal clock during the action-effect interval. This in turn leads to intentional binding, and links the effects of voluntary actions to the self.
\end{abstract}

\section{Introduction}

Distortions of the passage of time are commonplace: "time flies when you're having fun", but "the watched pot never boils".

One view attributes such distortions to modulation of a neural pacing signal, the "internal clock" (Treisman 1963; Gibbon et al. 1984; Matell and Meck 2004) that varies with stimulation and motor activity (e.g., Wearden et al. 1999). When clock rate decreases durations subjectively appear shorter because fewer internal time units accumulate in a given interval (e.g., Morrone et al. 2005). Conversely, when clock rate increases, durations seem longer (e.g., Hodinott-Hill et al. 2002).

Another view explains temporal illusions as recalibrations of the perceived onset time of sensory events. Predictable events, such as those produced by voluntary movements, may be pre-dated in order to ensure perceptual constancy (Yarrow et al. 2001), causality (Stetson et al. 2006), or sense of agency (Haggard et al. 2002).

Critically, recalibration theories account for temporal illusions as shifts by a constant interval of one timing stream relative to another, without changes in the rate of passage of time. Lag adaptation experiments support this possibility. For example, asynchronies between visual and auditory onsets, as in dubbed movies, soon become imperceptible, yet speaking rate appears normal (Fujisaki et al. 2004; Vatakis et al. 2007).

Many illusions involving modulation of subjective time fit either clock-rate explanations, time-shift explanations, or a hybrid of both. Here, we directly compared clock-rate and time-shift accounts of a temporal illusion known as 'intentional binding'. When a voluntary action is followed by a sensory effect (a tone), the action is perceived to occur later and the tone earlier than in control conditions with only 
actions, only tones, or involuntary TMS-induced movements plus tones (Haggard et al. 2002). People also perceive action-effect intervals as shorter than control intervals when estimating durations directly (Engbert et al. 2007, 2008).

In Experiment 1 participants made active or passive movements that were followed by tones after a short interval. Participants either estimated the interval duration [interval estimation (IE)], or judged whether two mild shocks delivered after action onset were successive or simultaneous (see Fig. 1).

Both clock-rate and time-shift theories predict shorter IE in the active than passive conditions, due to intentional binding. In contrast, the two accounts make different predictions regarding temporal discrimination (TD) of shocks during the action-effect interval (see Fig. 2 for model assumptions and predictions). If intentional binding reflects a slowed internal clock, then TD thresholds (TDTs) should increase following voluntary but not passive movements. A decrease in internal clock rate should produce an increase in TDTs because two shocks would be more likely to occur within a single, lengthened clock pulse.

Alternatively, clock slowing may be transient, rather than sustained throughout the action-effect interval. For example, if initial clock slowing is followed by a compen- satory acceleration of clock rate, then increased TDTs should be restricted to stimulation immediately after action, and the perception of interval duration should be relatively unaffected. Finally, if intentional binding is due only to time-shifts, without clock-rate change, TDTs should not differ between the two movement conditions. In addition, a control experiment (Exp. 2) investigated TD of shocks when active movements were not followed by tones. The purpose of Exp. 2 was to assess the potential impact of attentional lapses on TDTs that are caused by the demands of voluntary movement (e.g., Ruthruff and Pashler 2001).

\section{Methods}

\section{Participants}

Twenty paid right-handed subjects participated in Exp. 1 with local ethical committee approval. One participant was rejected, leaving 19 subjects (13 female, mean age, 24.6 years). For the excluded participant, the detection threshold procedure (see below) was unsuccessful, since she failed to detect even shocks that were $50 \%$ stronger

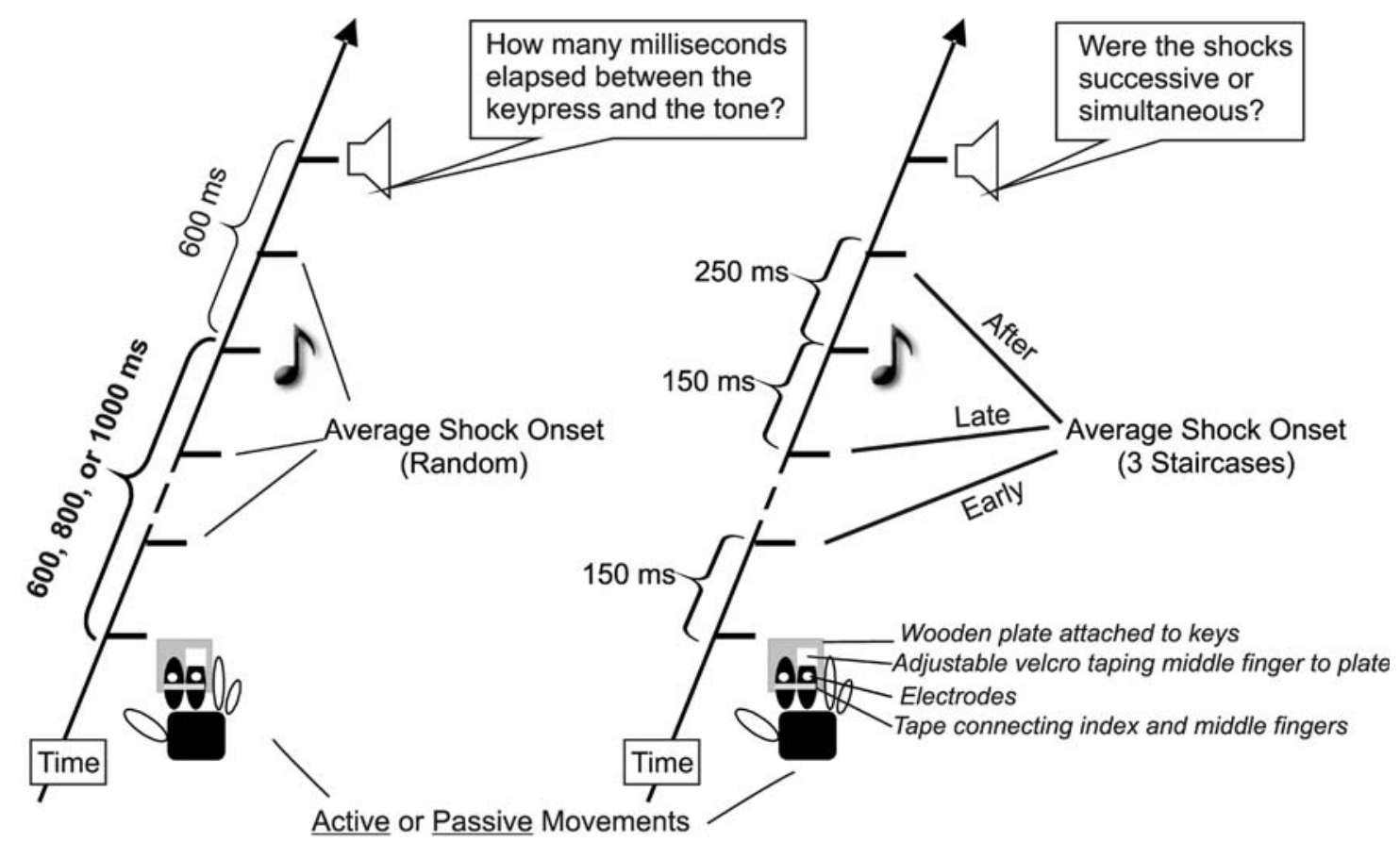

(a) Interval Estimation Trials

(b) Temporal Discrimination Trials

Fig. 1 Schematic illustration of trials in Exp. 1. Active and passive movements are followed by a tone after varying intervals. Two shocks are applied early or late within the action-effect interval, or after the tone. a On interval estimation trials participants estimate the interval between keypress and tone. Shock timing is determined by randomly drawing from one of the three staircases of the TD task. b On temporal discrimination trials the interval between the shocks is varied by a staircase procedure. Participants judge whether or not shocks are simultaneous. In Exp. 2 participants made active movements that were not followed by tones and always judged the simultaneity of the shocks 
(a) Re-calibration / Shift

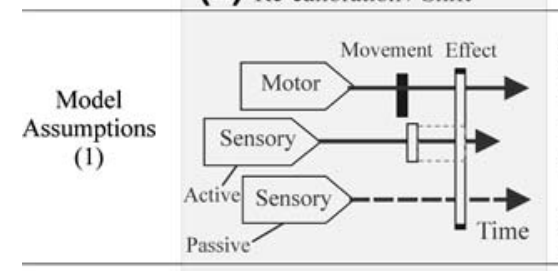

Predictions:

Binding

(2)
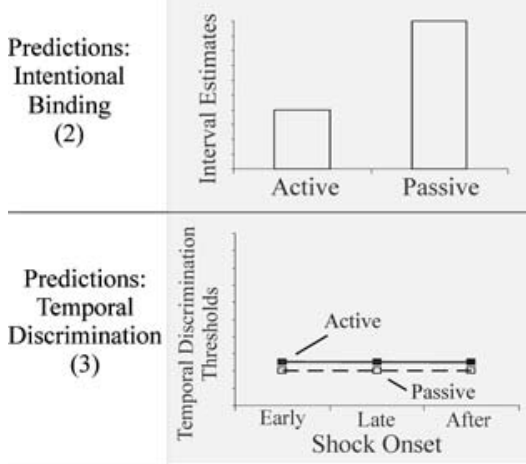

(b) Constant Clock Slowing

(c) Dynamic Clock Modulation
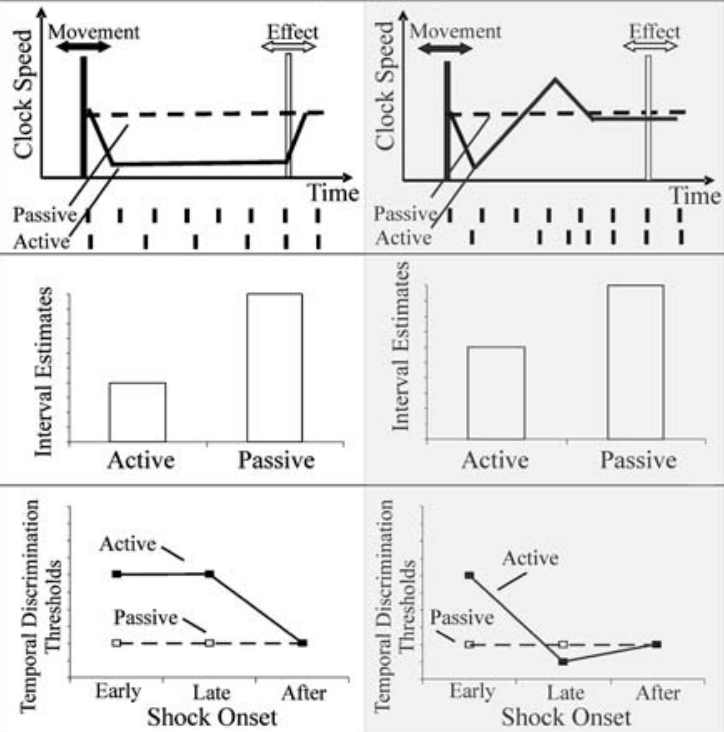

Fig. 2 Models of subjective time. a Recalibration/time-shift. (1) The perceived time of anticipated effects following voluntary movements is shifted backwards in time towards the action that caused them. This subjectively shortens action-effect intervals relative to passive control conditions (2), but clock speed remains unchanged, and so therefore does temporal discrimination (3). b Constant clock slowing. (1) Operant movements reduce clock speed throughout the action-effect interval. (2) Because fewer clock ticks accumulate, the action-effect interval appears shorter following active than passive movements. (3) Clock

than those used for other participants. ${ }^{1}$ Fourteen paid righthanded subjects (11 female, mean age 22.8 years) participated in Exp. 2.

\section{Design and procedure}

Subjects' right index and middle fingers were taped together, and a Velcro loop around the tip of the middle finger was attached to a wooden plate. Bipolar electrodes ( $4 \mathrm{~mm}$ diameter) were placed on the dorsal middle phalange of index and middle fingers (see Fig. 1). The electrodes were covered by the tape to ensure good skin contact. In the active movement conditions participants depressed a key beneath the wooden plate by moving down the taped fingers at a time of their choosing. They were asked to make discrete, fast movements in order to ensure maximum comparability between movement conditions and to limit variance in IE (see below). In the passive condition, the plate, and the fingers with it, was depressed by a

\footnotetext{
${ }^{1}$ At the time of testing, it was unclear whether this reflected an unusually conservative detection criterion, a failure to understand the instructions of the detection task, a particularly poor electrical interface between skin and electrodes, or a technical malfunction of the stimulator itself. Any of these explanations would have made continuing the experiment difficult and of doubtful scientifically value. Therefore, the experiment was discontinued.
}

slowing also impairs temporal discrimination during the action-effect interval, as the interval required to resolve successive stimuli is increased. c Dynamic clock modulation. (1) Initial clock slowing is followed by an increase of clock rate that partially compensates for the "lost" time. If compensation is underpowered, action-effect intervals seem shorter than after passive movements (2), but early impairments in temporal discrimination are followed by compensatory improvements (3)

servo motor a random $1,450-4,450 \mathrm{~ms}$ after trial start. Motor onset times were drawn from a reaction time distribution produced by two pilot subjects. The key and motor were shielded from participants' view. Active and passive movements were tested in separate blocks in counterbalanced order.

We first used a staircase procedure (Levitt 1971) to identify the intensity at which approximately $80 \%$ of shocks were detected on one electrode, $10 \mathrm{~ms}$ after a keypress. The electrode tested for detection (index or middle finger electrode) was counterbalanced across subjects. Intensity was varied by adjusting the stimulator pulse-width. Separate detection thresholds were found for active and passive keypresses, because active movements produce sensory attenuation (Williams et al. 1998). Experimental testing was performed at $135 \%$ of the estimated detection threshold in each condition, so that all shocks were clearly perceptible. A separate staircase was then used to adjust the intensity of the second electrode so it subjectively matched the first at the experimental level.

Each keypress (measured by a microswitch) produced a tone after an 600,800 , or $1,000 \mathrm{~ms}$ interval, chosen at random (Fig. 1). Tones were $70 \mathrm{~ms}$ in duration and $500 \mathrm{~Hz}$ in frequency. A prompt $600 \mathrm{~ms}$ after the tone either indicated to estimate the interval between keypress and tone in $\mathrm{ms}$ (IE trials), or to judge whether the two shocks were 
simultaneous or successive (TD trials). The trial types were chosen randomly and equiprobably. Thus, subjects could not predict whether they would judge the action-effect interval or shock simultaneity.

On TD trials, the interval between the shocks was controlled by a staircase procedure to estimate the temporal discrimination threshold (TDT). Three separate staircases were interleaved, for shocks immediately following the action (early stimulation time: Average shock $150 \mathrm{~ms}$ after keypress), late during the action-tone interval (average $150 \mathrm{~ms}$ before the tone, hence 450,650 or $850 \mathrm{~ms}$ after the action, for action-tone intervals of 600,800 or $1,000 \mathrm{~ms}$ ), and for stimulation after the tone ( $250 \mathrm{~ms}$ after tone onset). Shock onset was triggered by the keypress, as measured by microswitch. The three staircases were run in parallel, and trials were chosen from each staircase at random. The order of shocks (index finger then middle finger or vice versa) was also randomised. Each (descending) staircase started with an inter-stimulus interval of $300 \mathrm{~ms}$, sufficient for participants to reliably perceive the shocks as successive. Initial step size was $60 \mathrm{~ms}$, which was successively reduced by a factor of 3. Each staircase ended after a minimum of 4 reversals (Levitt 1971). TDT was defined as the mean intershock intervals of the third and fourth reversals.

On IE trials, participants made unspeeded verbal judgements of the duration of the interval between the moment that the key went down and the onset of the tone. They were told that interval length would range between $1 \mathrm{~ms}$ and $1,000 \mathrm{~ms}$, and were reminded that $1,000 \mathrm{~ms}$ correspond to $1 \mathrm{~s}$. Participants were encouraged not to restrict themselves to round numbers but instead to use the full numerical range. Before the experiment started subjects were provided with a "short" and a "long" reference interval for demonstration, and they received some practice with both IE and TD tasks alone, until they were familiar with the procedure. During the experiment itself, however, no reference interval was provided, nor was knowledge of results given at any stage. Shock timing on IE trials was determined by randomly drawing from one of the three staircases. At the end of each trial, the experimenter recorded the judgement (interval estimate or simultaneity judgement), and initiated the next trial by pressing the Enter key after asking "Ready?".

Exp. 2 differed from Exp. 1 in that (a) only active movements were required, (b) no tones were presented, and (c) the TD task was the only task.

\section{Results}

Interval estimates (see Fig. 3a) were analyzed using a $2 \times 3 \times 3$ ANOVA with movement condition (active, passive), action-effect interval (AEI; 600, 800, 1,000 ms), and
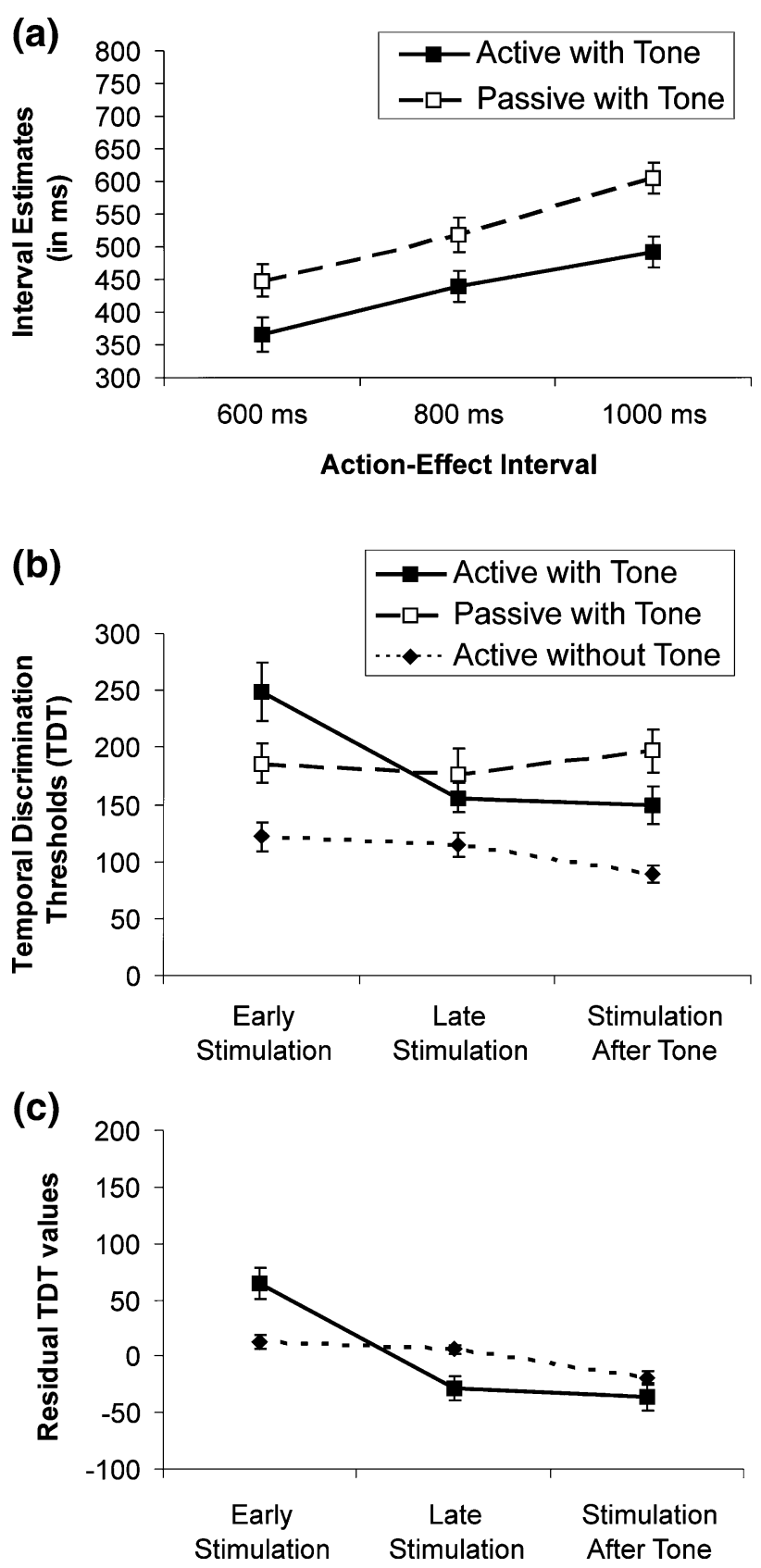

Fig. 3 Results (error bars show standard errors across individuals). a Mean interval estimates (in ms) according to movement condition and action-effect interval. b Mean temporal discrimination thresholds (TDTs) as a function of movement condition and time of stimulation. c Modulation of TDTs in the active movement conditions of Exps. 1 and 2. Overall differences between experiments have been removed, to show the residual modulation of TDT across stimulation times

stimulation time (early, late, after) as within subjects factors. Intervals were perceived as shorter with active than passive movements, $F(1,18)=23.3, P<0.01$. The main effect of action-effect interval was unsurprisingly significant, $F(2,36)=43.3, P<0.01$, indicating that participants were able to track the physical variation of the interval. However, the effect of interval did not interact with movement 
condition, $F(2,36)<1, P>0.4$. Finally, the average onset time of shock stimuli did not influence interval estimation (all effects involving stimulation time, $P$ 's $>0.15$ ).

Temporal discrimination thresholds (see Fig. 3b, c) were analyzed as follows.

First we compared the TDTs for the active and passive movement condition of Exp. 1 at the different stimulation times (early, late, after). This analysis yielded a significant main effect of stimulation time, $F(2,36)=7.99, P<0.01$, no significant effect of movement condition, $F(1,18)<1$, but a highly significant interaction between these factors, $F(2,36)=8.8, P<0.01$. The interaction was further analyzed by simple effect tests for both factors: TDTs differed across stimulation times for active movements, $F(2$, $36)=13.73, P<0.01$, but not for passive movements, $F(2,36)<1, P>0.5$, while active and passive conditions differed for early, but not late shocks: $t(18)=2.98$, $P<0.01, t(18)=-1.06, P>0.3$, with an additional difference after the tone, $t(18)=-2.36, P=0.03$. However, Bonferroni correction for three comparisons would give a critical $P$ value of 0.017 , suggesting caution regarding the comparison following the tone. We conclude that temporal discrimination was impaired immediately following operant actions, but reverted to the same level as passive movements later in the interval, and prior to the tone.

Second, to evaluate whether the intentional binding effect in the IE task of Exp. 1 was best explained by a sustained or by a temporary decrease in clock rate (Fig. 2b vs. c) we used multiple regression analysis to predict intentional binding (IE for active-passive movements) from TDTs. We transformed the TDT data into separate variables reflecting sustained and dynamic clock rate change, and used these variables to predict intentional binding. The total difference between active and passive TDTs in the action effect interval (i.e., the sum of the early and late stimulation differences between the active and the passive condition) was taken as an indicator of sustained change in clock rate, while the change between TDTs for early and late stimulation times (i.e., active minus passive TDT at early stimulation minus the active-passive difference at late stimulation) was taken to indicate dynamic clock rate change. The overall model with both predictors was significant, $F(2,16)=4.42, P<0.05$, but only dynamic clock-rate change predicted variance in intentional binding. The standardized weight for dynamic TDT change was $\beta($ dyn $)=0.6, t=2.96, \quad P<0.01$, whereas for sustained overall TDT it was $\beta$ (sus) $=-0.14, t=0.68, P>0.5$. This result suggests that intentional binding reflects dynamic changes of clock rate (Fig. 2c).

Finally, in order to determine whether the TDT modulation found for active movements in Exp. 1 was specific to operant actions, or due to attentional lapses associated with voluntary movements, we compared the TDTs following active operant movements (Exp. 1) with the TDTs for movements without tone-effects (Exp. 2). To this end we conducted a 2 (Experiment) $\times 3$ (stimulation time) mixed factors ANOVA on active movement TDTs. TDTs were generally lower in Exp. 2, $F(1,31)=16.2, P<0.01$, suggesting that omission of IE trials in that experiment may have allowed subjects to focus attention on the TD task, thus improving temporal discrimination. The main effect of stimulation time was also significant, $F(2,62)=13.88$, $P<0.01$, as was the interaction between experiment and stimulation time, $F(2,62)=5.94, \quad P<0.01$. Figure $3 \mathrm{c}$ shows the residual TDT values for each condition after removing the main effects of experiment, capturing the nature of this interaction more clearly. Residual TDTs were computed by subtracting participants' average TDT across stimulation times from their TDTs at each stimulation time. Simple effect tests were conducted to analyze the interaction further, and a Bonferroni correction for three comparisons was used, setting the $P$ value at 0.017 . The ANOVA of Exp. 2 TDTs with stimulation time as the only factor showed that TDTs were increased during early and late stimulation as compared to stimulation "after the tone", $F(2,26)=6.4, P<0.01$. More importantly, TDTs in the operant actions of Exp. 1 differed from those in the non-operant actions of Exp. 2: the residual TDT values after removing the main effect of experiment showed that TDTs were higher immediately after an operant action than after non-operant action $t(31)=-2.991, P<0.01$, but that this difference reversed for later stimulation $t(31)=2.8, P<0.01$. TDTs did not differ across experiments for stimulation after the tone $t(31)=-1.04, P>0.3$. This pattern of results suggests that a dynamic change in clock rate is specific to operant actions which produce a predictable effect, and does not occur as a simple by-product of motor action.

\section{Discussion}

Our study yielded three important results. First, we observed a large intentional binding effect in the IE task. Consistent with previous findings (Engbert et al. 2007, 2008), IE judgements that directly address the relation between actions and effects led to a larger and more robust effect than those typically observed with event timing judgements that require separate time judgements for actions and effects (e.g., Haggard et al. 2002). Intentional binding is unlikely to be due to differential interpretation of "movement" in the active and passive conditions because previous findings show that active movements are perceived as occurring earlier than passive movements when judged in isolation (e.g., Haggard et al. 2004). Second, our data revealed a clear deterioration of temporal discrimination 
immediately following voluntary actions, but not later in the action-effect interval. This deterioration was not due to a transient loss of attention caused by the demands of voluntary movement, because it was restricted to operant actions that predictably produced perceptual effects (Exp. 1), and was not found for identical actions without perceptual effects (Exp. 2). Third, the deterioration of temporal discrimination was correlated across subjects with subjective temporal compression of the action-effect interval. Importantly, these changes cannot be explained by the wellknown phenomena of sensory attenuation (Williams et al. 1998). First, we separately determined experimental shock intensities for active and passive movements, so shocks were equally detectable and salient across conditions. Thus, temporal discrimination impairment following active movements cannot be explained by impaired shock detectability due to sensory attenuation. Second, sensory suppression cannot explain the difference in temporal discrimination between experiments 1 and 2 .

Could these changes in time perception reflect division of attention? We should distinguish two senses of attention: dividing attention between tasks, and dynamically allocating attention to successive events. TDTs were indeed higher when subjects attended to both IE and TD tasks (Exp. 1), compared to TD alone (Exp. 2). Dividing attention between tasks might impair "on-line accumulation of temporal pulses" (Coull et al. 2004, p. 1506). However, divided attention cannot easily explain the interaction between tone occurrence and shock time. TDTs increased for shocks just after operant actions, with later shocks showing the reverse pattern. This interaction also rules out explanations based on dynamic allocation of attention. Making voluntary actions might produce a psychological refractory period (cf. Ruthruff and Pashler 2001; Tombu and Jolicoeur 2003), which could impair processing of shock events, thus increasing TDTs. The elevated TDTs following non-operant actions in Exp. 2 might reflect such an attentional effect. However, this refractory effect should be similar for actions with and without tones if transient changes in attention were the primary cause of TDT deterioration in the operant action condition. The statistical interaction between shock time and tone occurrence shows that this was not the case. Reduced attention to the interval as a consequence of dynamic allocation of attention to the expected tone cannot easily explain our results either, because active and passive movements in Exp. 1 produced comparable tones. Thus, our results cannot be attributed to attention alone.

Instead, the transient modulation of temporal discrimination in operant actions seems to reflect a genuine change in time perception. However, the changes in temporal discrimination cannot be explained simply by a time-shift effect induced by operant actions (Fig. 2a). Time shift theory proposes that the internal structure of time is unaltered. The core assumption is that the alignment between two clocks is adaptable, but the rate of each individual clock is not. Therefore the shift account can explain almost any change regarding when a single event is perceived to occur in relation to another. However, it cannot explain any change in TD ability within the adapted interval: Individual time slices or 'clock ticks' are thought to remain constant, which predicts comparable TDTs across conditions (see Fig. 2).

Rather, our data suggest that voluntary operant actions transiently slow down an internal clock, in anticipation of the effect of the action. Two shocks are thus more likely to fall within a single clock period, impairing temporal discrimination. In addition, clock slowing means that fewer clock cycles will occur between action and tone, producing the compression of the perceived interval between action and effect (i.e., intentional binding). This change in clock rate is quite brief: TDTs increased immediately following operant actions, but were not increased later in the actioneffect interval. Moreover, binding effects in IE were more strongly related to the dynamic change in TDTs across the action effect interval than to the average TDT level during the interval. Thus, the dynamic rate modulation model of Fig. 2c best accounts for our results. According to this model, initial clock slowing is followed by compensatory rebound acceleration. Rebound acceleration would have the effect of adjusting for "lost time" following the action (cf. Yarrow et al. 2001). However, our interval estimation data suggest that this compensation is incomplete. If initial clock slowing were exactly compensated by subsequent clock acceleration, there would be no net effect on perceived interval duration. Incomplete compensation, however, would produce temporal compression as in intentional binding. Incomplete compensation appears to be a general principle of many neural adjustments, including saccadic gain adaptation (e.g., McLaughlin 1967), and perceptual scaling (Taylor-Clarke et al. 2004). The nervous system may favor partial compensation over complete compensation or overcompensation on grounds of robustness.

Sustained slowing of an internal clock without any compensation (Fig. $2 b$ ) would produce a binding effect that increases with interval length. However, in our study, intentional binding was constant across intervals. This result implies that slowing was transient. It is consistent with our finding of reduced TDT later in the action interval, which implies a compensatory acceleration of an internal clock. Compensation for clock rate slowing occurred before the end of the shortest action-effect interval $(600 \mathrm{~ms}$ in our data). Our study randomised the action-effect interval, and did not estimate separate TDTs for each interval. Therefore, we cannot determine whether compensatory acceleration occurs within a fixed time window after action, or whether 
the compensation is predictively timed to occur just prior to the effect. In future research, we plan to use more predictable intervals, and check if temporal discrimination shows compensatory adjustment immediately before the predicted end of the interval.

We found that differences between conditions in time discrimination were consistent with differences in perceived interval duration. Moreover, changes in one measure were correlated across subjects with changes in the other. Not all timing illusions show this level of consistency. Stetson et al. (2007) found that the increased perceived duration of a whole-body motion (a $31 \mathrm{~m}$ free-fall) was not associated with any change in visual flicker fusion frequency. An important difference between their result and ours may be the involvement of voluntary action in our case, but passive displacement in theirs. We suggest that the cognitive motor system may predictively modulate subjective time in a coherent and consistent way as part of voluntary action. In contrast, the brain cannot plan ahead to guarantee a coherent experience of time for external sensory events such as passive movement. In this sense, the action system preconstructs a unified experience of subjective time, in contrast to our fragmented and revisable sensory experience of time (Stetson et al. 2007).

\section{Relation to other timing illusions}

Voluntary actions may cause both compression and dilation of subjective time. For example, in saccadic chronostasis (Yarrow et al. 2001) participants judge the duration of the first postsaccadic stimulus to be longer than subsequently presented reference stimuli. One explanation (Park et al. 2003) suggests that actions increase arousal, and thus internal clock rate. Our results cast doubt on this explanation. First, we found TDT increases after operant actions, implying reduced rather than increased clock speed. Second, our results show a compensatory dilation of subjective time that rapidly follows initial action-induced compression. Such dynamic changes are unlikely to reflect rapid and opposite modulations of arousal.

Morrone et al. (2005) recently reported underestimation of intervals between test stimuli immediately before a saccade. This subjective compression is similar to our IE effect. However, we could not easily test TDT before the self-paced actions of our study. In future work, we will test whether clock-rate slows anticipatorily during preparation of operant actions. A positive result would confirm the link between temporal discrimination and motor prediction (see below). Action may also reverse temporal order judgements (Morrone et al. 2005; Stetson et al. 2006). Impaired temporal discrimination would reduce the slope of the temporal order judgement function. However, previous studies reported action-related shifts in the function without slope changes. Hence temporal order effects may involve a timeshift mechanism, independent of clock-rate modulations.

Neural mechanisms of action-related time modulation

Classical timing models (e.g., Treisman 1963; Gibbon et al. 1984) involve an internal clock, an accumulator (memory for ticks), and a duration reference memory, against which the accumulator readout is compared. We have proposed that dynamic modulation of clock rates may occur during operant action. Neuropsychological, neuroimaging and neurointerventive evidence all suggest that the cerebellar cortex is involved in millisecond timing of sensory and motor events (for reviews see Ivry and Spencer 2004; Buhusi and Meck 2005). Arrival of parallel fibre action potentials at successive Purkinje cells' dendritic trees was originally viewed as cycling of an internal clock (Braitenberg 1967). However, parallel fibre firing represents instantaneous sensorimotor inputs to the cerebellum, whereas an internal clock should cycle continuously, like a pacemaker. Nevertheless, cerebellar activation in neuroimaging studies covaries with the interval between actions and effects (Blakemore et al. 2001). Moreover, cerebellar lesions interfere with the precise timing of predictive motor control in humans (Miall et al. 2007) and also with anticipatory responses to sensory events, such as conditioned eyeblinks (Perett et al. 1993; Gerwig et al. 2005). We therefore suggest that modulations of timing signals within the cerebellum could underlie both the compression of action-effect intervals, and action-related modulation of temporal discrimination, as part of a general process of sensorimotor prediction when preparing active voluntary movements. In contrast, a fronto-striatal network (cf. Buhusi and Meck 2005) may provide flexible timing for longer intervals based on oscillatory activity in distributed cortical circuits (Matell and Meck 2004).

Motor prediction and modulation of an internal clock

Current computational motor control models (Wolpert and Miall 1996; Bays and Wolpert 2007) suggest that the motor system predicts somatic and external effects of actions. Previous studies (e.g., Blakemore et al. 1998) showed that motor prediction leads to sensory attenuation of the predicted consequences of our actions. The pattern of timing distortions reported here may also reflect motor prediction. First, intentional binding at least partly reflects motor prediction (Moore and Haggard 2008). Second, our results show that intentional binding is related to clock slowing, and that timing distortions were absent both following passive movements, and following actions that did not produce predictable effects. Both findings suggest that modulations of neural timing mechanisms may occur as a consequence of motor prediction. 
Slowing an internal clock after action would boost the temporal contiguity between actions and effects, and also increase the likelihood of an effect occurring within a fixed number of clock cycles after action. That is, the prediction interval would become shorter, and less variable. Both influences would strengthen action-effect associations (Hommel et al. 2001), and give a strong basis for inferring causality (Hume 1748; Eagleman and Holcombe 2002). The term 'sense of agency' is sometimes (Haggard 2005) used to describe the feeling that we are in control of events. Agency may reflect a specific instance of causal inference in the case of one's own actions. Therefore, the modulations of neural timing reported here may contribute to computation of agency.

Acknowledgments This work was supported by ESRC grant RES000-23-1571, and by a Royal Society University Research Fellowship to Patrick Haggard. We would like to thank Matt Longo for helpful comments on this manuscript.

Open Access This article is distributed under the terms of the Creative Commons Attribution Noncommercial License which permits any noncommercial use, distribution, and reproduction in any medium, provided the original author(s) and source are credited.

\section{References}

Bays PM, Wolpert D (2007) Computational principles of sensorimotor control that minimize uncertainty and variability. J Physiol 578:87-396

Blakemore S-J, Wolpert DM, Frith CD (1998) Central cancellation of self-produced tickle sensation. Nat Neurosci 1:635-640

Blakemore S-J, Frith CD, Wolpert DM (2001) The cerebellum is involved in predicting the sensory consequences of action. Neuroreport 12:1879-1884

Braitenberg V (1967) Is the cerebellar cortex a biological clock in the millisecond range? Prog Brain Res 25:334-346

Buhusi C, Meck WH (2005) What makes us tick? Functional and neural mechanisms of interval timing. Nat Rev Neurosci 6:755-765

Coull J, Vidal F, Nazarian B, Macar F (2004) Functional anatomy of the attentional modulation of time estimation. Science 303:15061508

Eagleman DM, Holcombe AO (2002) Causality and the perception of time. Trends Cogn Sci 6:323-325

Engbert K, Wohlschläger A, Thomas R, Haggard P (2007) Agency, subjective time, and other minds. J Exp Psychol Hum Percept Perform 33:1261-1268

Engbert K, Wohlschläger A, Haggard P (2008) Who is causing what? The sense of agency is relational and efferent-triggered. Cognition 107:693-704

Fujisaki W, Shimojo S, Kashino M, Nishida S (2004) Recalibration of audiovisual simultaneity. Nat Neurosci 7:773-778

Gerwig M, Hajjar K, Dimitrova A, Maschke M, Kolb FP, Frings M, Thilmann AF, Forsting M, Diener HC, Timmann D (2005) Timing of conditioned eyeblink responses is impaired in cerebellar patients. J Neurosci 25:3919-3931

Gibbon J, Church RM, Meck WH (1984) Scalar timing in memory. Ann NY Acad Sci 423:52-77

Haggard P (2005) Conscious intention and motor cognition. Trends Cogn Sci 9:290-295
Haggard P, Clark S, Kalogeras J (2002) Voluntary action and conscious awareness. Nat Neurosci 5:382-385

Haggard P, Cartledge P, Dafydd M, Oakley DA (2004) Anomalous control: when 'free-will' is not conscious. Conscious Cogn 13:646-654

Hodinott-Hill I, Thilo KV, Cowey A, Walsh V (2002) Auditory chronostasis: hanging on the telephone. Curr Biol 12:1779-1781

Hommel B, Müsseler J, Aschersleben G, Prinz W (2001) The theory of event coding (TEC): a framework for perception and action. Behav Brain Sci 24:849-878

Hume D (1748) Enquiry concerning human understanding. PF Collier $\&$ Son, New York

Ivry RB, Spencer MC (2004) The neural representation of time. Curr Opin Neurobiol 14:225-232

Levitt H (1971) Transformed up-down methods in psychoacoustics. J Acoust Soc Am 49:467-477

Matell MS, Meck WH (2004) Cortico-striatal circuits and interval timing: coincidence detection of oscillatory processes. Cogn Brain Res 21:139-170

McLaughlin S (1967) Parametric adjustment in saccadic eye movements. Percept Psychophys 2:359-362

Miall RC, Christensen LOD, Cain O, Stanley J (2007) Disruption of state estimation in the human lateral cerebellum. PLoS Biology $5: 36$

Moore J, Haggard P (2008) Awareness of action: inference and prediction. Conscious Cogn 17:136-144

Morrone MC, Ross J, Burr D (2005) Saccadic eye movements cause compression of time as well as space. Nat Neurosci 8:950-954

Park J, Schlag-Rey M, Schlag J (2003) Voluntary action expands perceived duration of its sensory consequence. Exp Brain Res 149:527-529

Perett SP, Ruiz BP, Mauk MD (1993) Cerebellar lesions disrupt learning-dependent timing of conditioned eyelid responses. J Neurosci 13:1708-1718

Ruthruff E, Pashler H (2001) Perceptual and central interference in dual-task performance. In: Shapiro K (ed) The limits of attention: temporal constraints in human information processing. Oxford University Press, New York, pp 100-123

Stetson C, Cui X, Montague PR, Eagleman DM (2006) Motor-sensory recalibration leads to an illusory reversal of action and sensation. Neuron 51:651-659

Stetson C, Fiesta M, Eagleman DM (2007) Does time really slow down during a frightening event? PLoS ONE 2:1295

Taylor-Clarke M, Jacobsen P, Haggard P (2004) Keeping the world at a constant size: object constancy in human touch. Nat Neurosci 7:219-220

Tombu M, Jolicoeur P (2003) A central capacity sharing model of dual task performance. J Exp Psychol Hum Percept Perform 29:3-18

Treisman M (1963) Temporal discrimination and the indifference interval: implications for a model of the "internal clock". Psychol Monogr 77:1-31

Vatakis A, Navarra J, Soto-Faraco S, Spence C (2007) Temporal recalibration during asynchronous audiovisual speech perception. Exp Brain Res 181:173-183

Wearden JH, Pilkington R, Carter E (1999) 'Subjective lengthening' during repeated testing of a simple temporal discrimination. Behav Processes 46:25-38

Williams SR, Shenasa J, Chapman CE (1998) Time course and magnitude of movement-related gating of tactile detection in humans I: importance of stimulus location. J Neurophysiol 792:947-963

Wolpert DM, Miall RC (1996) Forward models for physiological motor control. Neural Netw 9:1265-1279

Yarrow K, Haggard P, Heal R, Brown P, Rothwell JC (2001) Illusory perceptions of space and time preserve cross-saccadic perceptual continuity. Nature 414:302-305 